\title{
DIAGNÓSTICO DE ENFERMAGEM UM DESAFIO DE ENFERMAGEM PARA OS ANOS 90 *
}

\author{
Vera Lúcia de Almeida Bezerra Perez ** \\ Maria Miriam Lima da Nóbrega $* * *$ \\ Juracy Nunes de Farias **** \\ Marga Simon Coler *****
}

\begin{abstract}
RESUMO - Os autores deste trabalho apresentam um relato histórico dfo desenvolvimento dos diagnósticos de enfermagem, incluindo definições e Sistemas de Classificação. É discutida a evolução a partir de uma lista alfabética de nomes de diagnósticos para os Padrões de Respostas Humanas da NANDA (North American Nursing Diagnosis Association). O desafio surge da necessidade de participação internacional na validação, notificação e conseqüente estabelecimento da Taxonomia para a aceitação no CID-10 (Código Internacional de Doenças).
\end{abstract}

\begin{abstract}
The authors of this paper present a historical account of the development of Nursing Diagnossis, including definitions and the classification systems. The evolution from an alphabetized list of disgnostic labels to the Human Response Patterns of NANDA (North American Nursing Diagnosis Association) is discussed. The Challenge Arisses in the necol for international participation in the validation, modification and Consequent stabilization of the Taxonomy, as it heads for acceptance in the CID 10 (International Code of Diseases)
\end{abstract}

\section{INTRODUÇÃO}

A Enfermagem sempre enfrentou desafios para se firmar como ciência e profissão. Entre outros, a aplicação do processo de enfermagem, como o instrumento ou método profissional para guiar sua prática, foi o que mais movimentou os enfermeiros no sentido de adquirir uma autonomia profissional, para concretizar a proposta de promover, manter ou restaurar o nivel de saúde do cliente.

Nas duas últimas décadas, verificou-se um reconhecimento da importância e da necessidade do diagnóstico de enfermagem, numa tentativa de uniformizar e padronizar a terminologia utilizada pelos enfermeiros para referir os problemas do cliente pelos quais têm responsabilidade profissional.

Segundo GORDON ${ }^{6}$, diagnóstico de enfermagem é o termo usado para determinar a súmula dos dados e descrever os problemas de saúde, atual ou potencial, do cliente. $O$ diagnóstico

a avaliação clínica dos problemas de saúde do cliente feito pelo enfermeiro.

O desenvolvimento do diagnóstico de enfermagem teve seu início de várias maneiras. Surgiu na literatura em $1950 \mathrm{com}$ MACMANUS ${ }^{4}{ }^{5}{ }_{7} 11$, passando na década de 60 a integrar as fases do processo de enfermagem ${ }^{6}$ e só passou a ser utilizado na prática clínica da enfermagem depois da $1^{\mathrm{a}}$ Conferencia do Grupo de Diagnósticos de Enfermagem, em 1973, que passou a denominar-se Associação Norte Americana de Diagnósticos de Enfermagem (NANDA-North American Nursing Diagnosis Association), em $1982^{5}$ e está se expandindo por diversos países numa tentativa internacional de padronização dos diagnósticos de enfermagem.

Este desafio de internacionalizar o desenvolvimento dos diagnósticos de enfermagem é uma questão profissional concernente a todos os enfermeiros, para permitir a aceitação universal da sua terminologia, a continuação da classificação sobre as condições de saúde do cliente, o que favorecerá a diminuição de erros na comunicação e a construção de outros sistemas de classificação, o que beneficiará a prática, o ensino e a pesquisa em enfermagem 5 .

Considerando que os diagnosticos de enfermagem representam, no momento, um desafio internacional para a nossa profissão e que há falta de consenso entre os enfermeiros quanto ao significado deste termo, pretendemos com este trabalho apresentar uma retrospectiva histórica e o desenvolvimento do diagnostico de enfermagem, bem como despertar os enfermeiros para o espaço que terá a Enfermagem Brasileira neste desafio.

\footnotetext{
* Prémio Noraci Pedrosa - 3 Lugar - 41 Congresso Brasileiro de Enfermagem - Florianópolis-SC

** Professor Adjunto II do Departamento de Enfermagem de Saúde Pública e Psiquiatria da UFPB

*** Prof. Adjunto I do Departamento de Enfermagem de Saúde Pública e Psiquiatria da UFPB

**** Prof. Adjunto I do Departamento de Enfermagem Médico-Cirúrgica e Administração da UFPB

***** Professor Doutor da Escola de Enfermagem de Connecticut-Estados Unidos
} 


\section{DESENVOLVIMENTO DO DIAGNÓSTICO DE ENFERMAGEM}

\subsection{Histórico}

O termo diagnóstico de enfermagem tem uma historia relativamente curta, encontrando seus passos iniciais no advento da enfermagem moderna, quando NIGHTINGALE e suas colegas diagnosticaram problemas de saúde, nos soldados, na Guerra da Criméa ${ }^{6}$.

Quase um século depois, na década de 50, os enfermeiros resgataram a experiência de NIGHTINGALE, com o objetivo de planejar a prática profissional e educacional da enfermagem. Mas a tentativa desses enfermeiros trazia consigo a preocupação de que "diagnóstico" é um termo que expressa uma atribuiçãoo do médico ${ }^{6}$.

A partir de então, inúmeros trabalhos sobre diagnostico de enfermagem foram feitos, e reconheceram a importância de uma avaliação clínica e da identificação dos problemas de saúde que requerem interveções de enfermagem, mas até que esta terminologia chegasse a ser usada na prática clínica, teve que ser desenvolvida com uma linguagem clara e consistente ${ }^{5}$

GORDON $^{6}$ afirma que nos anos 60 LEVINE sugeriu a palavra "TROPHIGNOSIS" para tornar clara a idéia do diagnóstico de enfermagem. Outros, no entanto, acreditavam ser o termo diagnóstico de enfermagem mais facilmente entendido, e em 1967 KING dissipou a noção de que apenas o médico é quem usa o processo diagnostico. Atualmente o termo diagnóstico de enfermagem é usado em jornais, livros texto e manuais, para descrever problemas de saúde do cliente identificados pelo enfermeiro.

Em 1973, um grupo de enfermeiros reuniu-se em St. Louis e organizou a $1^{\text {a }}$ Conferência Nacional para Classificação de Diagnosticos de Enfermagem. Nessa ocasião, os enfermeiros iniciaram o esforço formal de identificar, desenvolver e classificar os Diagnósticos de Enfermagem. A partir dessa conferência já se realizaram sete, nas quais os participantes desenvolveram e identificaram o termo diagnóstico para descrever os problemas de saúde, atual ou potencial, tratáveis pelas intervenções de enfermagem.

Em 1982, a Conferência foi aberta à comunidade de enfermagem, onde a NANDA foi formalmente organizada e seu Regimento Interno, aprovado.

A participação da Enfermagem Brasileira no desenvolvimento do diagnóstico de enfermagem a nível internacional ocorreu em 1988 com a apresentação de um estudo $^{3}$ na $8^{\text {a }}$ - Conferência da NANDA sobre diagnostico de Enfermagem. A partir de então têm sido desenvolvidos estudos sobre diagnósticos de enfermagem na Universidade Federal da Paraíba, como testemunho de engajamento neste desafio.

Atualmente o Comitê de taxonomia da NANDA e representantes da Associação Americana de Enfermagem (ANA-American Nurses Association), desenvolveram o Código dos Diagnósticos de Enfermagem, propondo a sua inclusão pela Organização Mundial de Saúde-OMS, na Classificação Internacional de Doenças-CID $10^{10}$.

Concominantemente a NANDA continua os estudos no seu proposito de "desenvolver, refinar e promover uma Taxonomia da terminologia dos diagnósticos de enfermagem para uso geral dos enfermeiros" e está elaborando a 1ํ Versão da Taxonomia II que será apresentada na 9ª Conferência em $1990^{10}$.

\subsection{Definição de Diagnóstico de Enfermagem}

Segundo GORDON ${ }^{6}$, diagnóstico de enfermagem pode ser definido como categoria e como processo. Como categoria pode ser visto em três perspectivas: idéia;

1) conceitual, que comunica o significado de uma

2) estrutural, que descreve as várias formas de registro dos diagnosticos; e

3) contextual, que descreve especificamente a relação do diagnóstico com o processo de enfermagem e os cuidados de saúde. Como processo o diagnóstico apresenta duas perspectativas:

refere-se ao nome dado aos problemas de saúde no sistema de classificação do diagnóstico de enfermagem; e

a avaliação clínica.

\subsubsection{Definição do Diagnóstico de Enfermagem como Categoria:}

\subsubsection{Definição Conceitual:}

A Definição Conceitual transmite o significado de uma idéia e apresenta dua importantes características:

1) a responsabilidade de se fazer diagnostico de enfermagem é exclusiva do enfermeiro; e

2) descreve os problemas de saúde atual ou potencial do cliente. entende-se por problema de saúde atual, o desvio da linha da saúde e a identificação dos fatores de riscos os quais decorrem de uma predisposição individual, familiar ou comunitária. E por problema de saúde potencial, a presença dos fatores de riscos.

Em outras palavras, o diagnбstico de enfermagem é o julgamento clínico, feito pelo enfermeiro, para descrever os problemas de saúde, atual ou potencial, do cliente.

\subsubsection{Definição Estrutural:}

A Definição Estrutural é constituída pelos problemas de saúde, fatores etiologicos e definição de características ou grupos de sinais e sintomas os quais são referenciados pela sigla $\mathbf{P E S}^{6}$.

Nesta definição, 0 primeiro componente do diagnóstico de enfermagem são os problemas de saúde, os quais são ilustrados pelas categorias diagnosticas. Cada uma descreve o problema ou estado de saúde do indivíduo, famnlia ou comunidade, o qual é expressado de forma clara, com termos resumidos, preferentemente por duas ou três palavras.

$\mathrm{O}$ segundo componente do diagnóstico de enfermagem são os fatores prováveis que causam ou mantém os problemas de saúde do cliente. Estes po- 
dem ser, comportamentos dos clientes e fatores ambientais, ou a interação de ambos. $\mathrm{O}$ cliente pode ter alguns problemas, mas existem sinais e sintomas indicativos de dife $e_{i}$ entes fatores etiologicos. Isto $\varepsilon$ importante para realizar a diferença diagnostica existente, como tambem identificar o tratamento requerido, quando os fatores causais são diferentes.

O terceiro componente são os sinais e sintomas os quais incluem a condição de critérios, na definição das características e no suporte das informações. Quando o cliente manifesta sinais e sintomas que correspondem ao critério de definição de características, eles são usados na categoria diagnostica apropriada. Isto irá favorecer a padronização e utilização internacional dos diagnosticos de enfermagem.

\subsubsection{Definição Contextual:}

Na Definição Contextual o diagnóstico de enfermagem É examinado no seu lugar correto, ou seja, dentro do processo de enfermagem $\varepsilon$ prover um foco para o planejamento e avaliação do cuidado de enfermagem, e isto só será possível com a utilização do processo de enfermagem.

\subsubsection{Definição do Diagnóstico de Enfermagem como Processo}

A palavra diagnostico e percebida previamente tendo dois significados. $\mathrm{O}$ primeiro refere-se ao nome para os problemas de saúde que se encontram no sistema de classificação de diagnóstico de enfermagem. $\mathrm{O}$ segundo refere-se ao processo que leva a avaliação clínica.

O processo diagnóstico e essencialmente uma maneira de avaliar fatores etiologicos que influenciam os estados de saúde do cliente.

Diagnosticar e distribuir ou discriminar. As informaçōes são coletadas e a primeira discriminação que ocorre $\varepsilon$ entre indicadores de problemas e indicadores de saúde. Esta $\varepsilon$ uma tarefa discriminatória com dois caminhos. Se os sinais e sintomas apontam em direção ao julgamento clínico de saúde, eles são descritos e o reconhecimento $\varepsilon$ dado para as práticas de promoção da saúde do cliente. A identificação e o reconhecimento das práticas da promoção da saúde servem para reforçar o comportamento que irá contribuir para a manutenção da saúde.

Por outro lado, se os sinais e sintomas apontam na direção dos problemas de saúde, atual ou potencial, a busca de estratégias são necessárias para identificar claramente o problema. Adicionalmente o conjunto de sinais e sintomas deve ser classificado corretamente como uma categoria diagn6sticada. Este processo representa muito mais que uma diferenciaçấo entre dois caminhos que são a presença ou a ausência de saúde.

Segundo GORDON ${ }^{6}$, para se entender diagnostico de enfermagem como categoria e processo é necessário saber que:

- diagnósticos de enfermagem são feitos por enfermeiros;

- diagnosticos de enfermagem descrevem os problemas de saúde atual ou potencial, que sảo amenizados pelas intervenções de enfermagem;

- os diagnósticos de enfermagem sẩo nomes que refe- rem o conjunto de sugestões indicando o problema de saúde;

- cada diagnбstico de enfermagem tem um pequeno conjunto de características definidas, que representam os sinais e sintomas dos problemas de saúde. Estas características representam o critério diagnostico para fazer o diagnóstico;

- cada diagnóstico de enfermagem tem etiologia ou fatores relacionados que contribuem para manter $o$ problema; sua resolução ou modificação serve como foco para a intervenção de enfermagem. Neste caso, os problemas potenciais e fatores de riscos são identificados;

- dentro do processo diagnóstico existem a coleta de informaçōes, interpretação das informações e a denominação dos diagnosticos.

\subsection{Desenvolvimento do Sistema de Classificação}

O primeiro sistema de classificação relevante para a prática e para os objetivos terapéuticos da enfermagem, foram os 21 problemas listados por ABDEL$\mathrm{LAH}$, em $1957^{\circ}$ ?.

O desenvolvimento do atual sistema de classificação de diagnosticos de enfermagem teve seu ponto de partida na 1 $1^{\mathrm{a}}$ Conferência do Grupo de Diagnosticos de Enfermagem em 1973, onde os diagn6sticos de enfermagem apresentados foram classificados em ordem alfabetica ${ }^{5}$.

Desde então foi constituído um grupo de enfermeiras teoristas, com o objetivo de criar um sistema de classificação de diagnósticos de enfermagem. Em 1977, ROY, como membro participante deste grupo, convenceu os demais teoristas da necessidade de desenvolver uma estrutura para a organização dos diagnósticos no sistema de classificação; elaborar recomendações sobre a abstração dos níveis diagnósticos; correlacionar os trabalhos do grupo ao progresso do desenvolvimento das categorias e esclarecer a relevância da estrutura para a prática de enfermagem ${ }^{6}$.

Parelamente e esse estudo, GORDON, que tambem era membro do grupo de teoristas, introduziu no início da década de 80, os 11 Padrões Funcionais da Saúde (vêr Quadro II) os quais serviram para desenvolver os diagnósticos de enfermagem, a partir da lista alfabética e de outros istemas diagnósticos, para um método de classificação amplamente utilizado, o qual foi adotado pelo Grupo ${ }^{3}$.

\section{Padrões Funcionais de Saúde}

- Padrão de percepção e manutenção da saúde

- Padrão nutricional metabolico

- Padrão de eliminação

- Padrão de atividade e exercício

- Padrão de sono e repouso

- Padrão perceptual cognitivo

- Padráo de autopercepção e autoconceito

- Padráo de relacionamento de papés

- Padrão sexual reprodutivo

- Padrão de tolerancia ao stress

- Padráo de crenças e valores

Os Padrỏes Fancionais de Saúde envolvem a interaçảo do cliente e o meio ambiente e refletem a sequéncia de comportamentos na área da vida humana, e são análogos: ao sistema orgânico que é usado pelo 
médico para fazer a anamnese.

Nos seus relatorios subsequentes, o grupo de teorististas apresentou uma estrutura para o sistema de classificação dos diagnósticos de enfermagem com base no conceito holístico do ser humano para abordar a saúde, estrutura esta denominada "Nove Padrões de Unidades do Homem".

Quando a NANDA foi organizada, em 1982, o grupo de teoristas foi desfeito, sendo criado o Comitê de Taxonomia presidido por PHYLLIS KRITEK, com o objetivo de desenvolver o Sistema de Classificação para os diagnósticos de enfermagem.

Em 1986, a Taxonomia I da NANDA foi aprovada em Assembléia Geral e os termos Padrões de Respostas Humanas foram criados para substituir os Nove Padrōes de Unidade do Homem constituindo assim, oficialmente, a estrutura conceptual para a organização da Taxonomia I, que trataremos a seguir.

\begin{tabular}{|l|}
\hline Padrões de Respostas humanas \\
\hline - TROCANDO - envolvendo, dar e receber mutua- \\
mente \\
- COMUNICANDO - envolvendo o envio de men- \\
sagens \\
- RELACIONANDO - envolvendo o estabelecimen- \\
to de vínculos \\
- VALORIZANDO - envolvendo atribuições de um \\
valor relativo \\
- ESCOLHENDO - envolvendo a seleção de alterna- \\
tivas \\
- MOVENDO - envolvendo atividades \\
- PERCEBENDO - envolvendo a recepção de infor- \\
mações \\
ciado com a informação \\
CONHECENDO - envolvendo o significado asso- \\
da informação
\end{tabular}

\subsection{Desenvolvimento da Taxonomia dos Diagnósticos de Enfermagem}

O esforço internacional no desenvolvimento da Taxonomia dos diagnósticos de enfermagem, provém da prática clínica, da pesquisa e da educação na enfermagem ${ }^{8}$.

A Taxonomia é um termo usado como sinônimo de classificação, mas refere-se também à ciência da classificação. Na enfermagem ela provém dos Padrões de Respostas Humanas da NANDA e consiste em ordenar os diagnosticos de acordo com a relação prática. Para isto é necessário que tenha: consistência, 16gica, precisão, objetividade, neutralidade, compreensão e margem para a introdução de outros diagnósticos ${ }^{2}$

A Taxonomia I da NANDA está formada por cinco níveis representando uma ordem hierárquica de acordo com o nível de abstração. Os Padrões de Respostas Humanas constitui o Nível I, que é o nível mais abstrato e a estrutura conceptual para a organização da Taxanomia que se torna concreta na sua descendência vertical ${ }^{2}$ :

O Nivel II representa as alterações nas subcategorias dos padrões. O Nível III descreve o fenômeno da preocupação e os níveis IV e $V$ são os mais concretos, os quais contêm as correntes diagnosticas e são usados para determinar a assistência de enfermagem.
A NANDA tem desenvolvido um ciclo formal de revisão de Diagnбstico de Enfermagem, assegurando contínuo desenvolvimento e refinamento dos mesmos e possibilitando a inclusão de novos diagnósticos submetidos por partes interessadas.

O Código de Diagnóstico de Enfermagem proposto pela NANDA e representantes da ANA, para inclusão, pela OMS, no CID $^{10}$ apresenta apenas os níveis I e II da Taxonomia I Revisada ${ }^{10}$. Mas, para efeito de estrutura e utilização na prática clínica permanecem os cinco níveis já descritos anteriormente.

\section{QUADRO IV}

\begin{tabular}{|ll|}
\hline TAXONOMIA I DA NANDA \\
\hline Nível I & - 9. Sentido \\
- Nivel II & - 9.1 Alteração no conforto \\
- Nivel III & - 9.1.1 Dor \\
- Nivel IV & - 9.1.1.1 Crônica \\
- Nivel II & - 9.2 Alteração na Integridade Emocional \\
- Nível III & - 9.2.1 Reação de Pesar \\
- Nivel IV & - 9.2.1.1 Disfuncional \\
& - 9.2.1.2 Antecipatoria \\
- Nivel III & - 9.2.2 Potencial para violência \\
& - 9.2.3 Resposta pós-trauma \\
- Nivel IV & - 9.2.3.1 Síndrome ao trauma de estupro \\
- Nivel V & - 9.2.3.1.1 Reação composta \\
- Nível V & - 9.2.3.1.2 Reação silenciosa \\
- Nivel II & - 9.3 Alteração no estado emocional \\
- Nivel III & - 9.3.1 Ansiedade \\
& - 9.3.2 Medo \\
\hline
\end{tabular}

Taxonomia revisadas em junho de 1988 (9).

\section{QUADRO V}

PROPOSTA DA VERSÃO DA TAXONOMIA I
DA NANDA, REVISADA PARA O
CID10: CONDIÇÕES QUE NECESSITAM
CUIDADOS DE ENFERMAGEM

PADRÃO DE RESPOSTA HUMANA: ESCOLHENDO

- 00 Lidar familiar enfraquecido

00.0 Descompromissado

00.1 Incapacitado

- 01 (Comportamento para elevar o nível de saúde)

01.0.9. Comportamentos para elevar o nível de saúde (especificar)

- 02 Lidar individual enfraquecido

02.0 Ajustamento enfraquecido

02.1 Conflito de decisão

02.2 Lidar defensivo

02.3 Negação enfraquecido

02.4 Recusa

\section{CONSIDERAÇÕES GERAIS}

Considera-se o Diagnóstico de Enfermagem de vital importância para a profissão em virtude de permitir a universalização da terminologia científica da enfermagem e uma base unificada para a decisão sobre os cuidados requeridos pelo cliente sob a responsabilidade do enfermeiro. Isto fortalecerá o desenvolvimento de pesquisas sobre os cuidados de enfermagem e concretizará cada vez mais o saber da enfermagem.

Considerando-se que a Taxonomia I da NANDA 
pode ser utilizada independentemente da sistematização ou teorização adotada para o processo de enfermagem, e que existem no Brasil vários núcleos de estudos e esforços para introduzir efetivamente o processo de enfermagem na prática clínica, sugere-se que:

- seja divulgado mais amplamente entre os enfermeiros, o desenvolvimento do sistema de classificação dos diagnosticos de enfermagem;

- sejam constituídos grupos de estudo sobre os diagnósticos de enfermagem a nível nacional, regional e local, para que, desta forma, se de- senvolva o processo de enfermagem com todas as suas etapas, utilizando-se o sistema de classificação dos diagnósticos de enfermagem;

- torne-se um dos temas oficiais para estudo pelas Comissões de educação e pesquisa da ABEn.

Espera-se, portanto, uma resposta dos enfermeiros, a partir deste congresso, para ocupar seu espaço neste desafio de universalizar os diagnosticos de enfermagem.

\section{REFERÊNCIAS BIBLIOGRÁFICAS}

1 CAMPEDELLI, M. C. et alil Processo de Enfermagem na Prática. São Paulo: Ática, 1989.32p

2 COLER, M. S. et alil. NANDA Taxonomy I: A Preliminary Validation/Invalidation Study. In: Nursing Scan in Reserarch: Application for Clinical Practice. Philadephia. Lippincott, v. 1, no 4 Nov/Dez, 1988. p. 1 - 4.

3 COLER S.M., PÉREZ, V. L. A. B. \& NOBBREGA, M.M.L. Justification for Mental Health Services at the Departament of Nursing Universidade Federal da Parara Brasil. Through the Utilization of Nursing Diagnosis and Diagnostic Categories. In: CARROLL JOHNSON, R.M. Classification of Nursing Diagnosis: Proceedings of the Eighth Conference. Philadelphia: Lippincott, 1989. p. 165-169.

4 COLLER, M.S. \& VICENT, K.G. Coged Nursing Diagnosis on Axes. A Prioritized, Computer - Ready Diagnostic System for Psychiatric/Mental Health Nurses. In: Archives of Psychiatric Nursing. v. 1, m.2 April, 1987. p. 125-131.

5 FREDE T TE, S. LaFortune \& O'NEILL, E. Sjoberg. Can theory improve diagnosis? An examination of the relatio-ship between didactic content and the ability to diagnose in clinical practice. In: MCLANE, A.M. Classification of Nursing Diagnosis: Proceedings of the Seventh Conference. St. Louis: Mosby Company, 1987. p. 423

6 GORDON, Marjory. Nursing Diagnosis: Process and Application. 2 ed., New York: McGraw-Hill, 1987.
7 McKEIGEEN, Rosemary J. et alii. Nursing diagnosis: assessment of use in acute care settings in Iowa. : McLANE, A.M. Classification of Nursing Diagnosis - Proceedings of the Seventh Conference. St. Louis: Mosby Company, 1987.p. 400.

8 McLANE, A.M. \& KIM, M. J. Integration of Nursing Diagnoses in Curriculo of Baccalaureate and Graduate Programas of Nursing: A urvey. In: CARROL JONHSONm R. M. Classification of Nursing Diagnosis - Proceeding of the Highth Conference. Philadelphia, Lippincott, 1989.p.50.

9 NANDA Taxonomy I Revised (June 1988). In: CARROL JOHNSON, R.M. Classification of Nursing Diagnosis: Proceeding of the Eighth Conference. Philadelphia: Lippincott, 1989. pp. 511-514.

10 NANDA, Taxonomy I Revised with Official Diagnostic Categories. St. Louis, 1989.p. 121-122.

11 ROSSI, Laura. Organizing data for nursing diagnoses using functional health patterns. In: McLANE, A.M. Classification of Nursing Diagnosis: Proceedings of the Sevenht Conference. St. Louis: Mosby Company, 1987. p.97.

12 WERLEY, Harriet. Nursing Diagnosis and Nursing minimum data set. In: McLANE, A.M. Classification of Nursing Diagnosis: Proceedings of the Seventh Conference. St. Louis, Mosby Company, 1989. p.22. 